\title{
Musculoskeletal care in haemophilia: the importance of the joint team approach
}

\author{
Haroon Mann, Paul McLaughlin
}

The multidisciplinary team approach to providing comprehensive care is the cornerstone of the management of musculoskeletal issues in haemophilia. At the Royal Free the team takes a holistic approach to musculoskeletal disease management that is not just about assessment of a painful knee or having an operation. It recognises that patients sit on a spectrum of musculoskeletal disease severity that is dependent on age and the type of treatments available and the issues that may have been experienced with those treatments. The patient's own goals sit at the heart of the team's approach.

Keywords: orthopaedic, haemophilia, Katharine Dormandy, physiotherapy, joints

\section{The surgeon's perspective...}

Orthopaedic surgery became a sub-specialty after the Second World War but it was not until the 1980s when arthroplasty and implant surgery were introduced that the orthopaedics department at the Royal Free Hospital began to develop a reputation. As the patient cohort grew and the workload increased, surgeons such as Mr Colin Madgwick were faced with increasingly complex presentations in severe haemophilia patients, as a consequence largely of joint bleeds, including arthropathy of the joints other than hips and knees. Figure 1 shows a patient with an ankylosed knee joint. This was a common presentation in the 1980s, but is now rarely seen in the UK because of the treatments we use.

The challenge today remains the destructive and debilitating nature of haemophilia and the immobility it can cause people in the prime of their life. These are young people who want to go out and play football and participate in all the normal activities that young people want to do. But it is difficult because their ankles, their elbows, their knees, are all affected. Joint problems in haemophilia affect young people's education, their careers, their financial security and their general quality of life. Patients come to the centre and want to get back to football and actively participating in their lives.

We have no magic bullet for when such problems arise

Haroon Mann, Consultant Trauma \& Orthopaedic Surgeon and Honorary Senior Lecturer, Royal Free Hospital and University College Medical School, London. E-mail: mann.ha@nhs.net

Paul McLaughlin, Clinical Specialist Physiotherapist in Haemophilia, Katharine Dormandy Haemophilia Centre, Royal Free Hospital, Pond St, London. E-mail: p.mclaughlinanhs.net

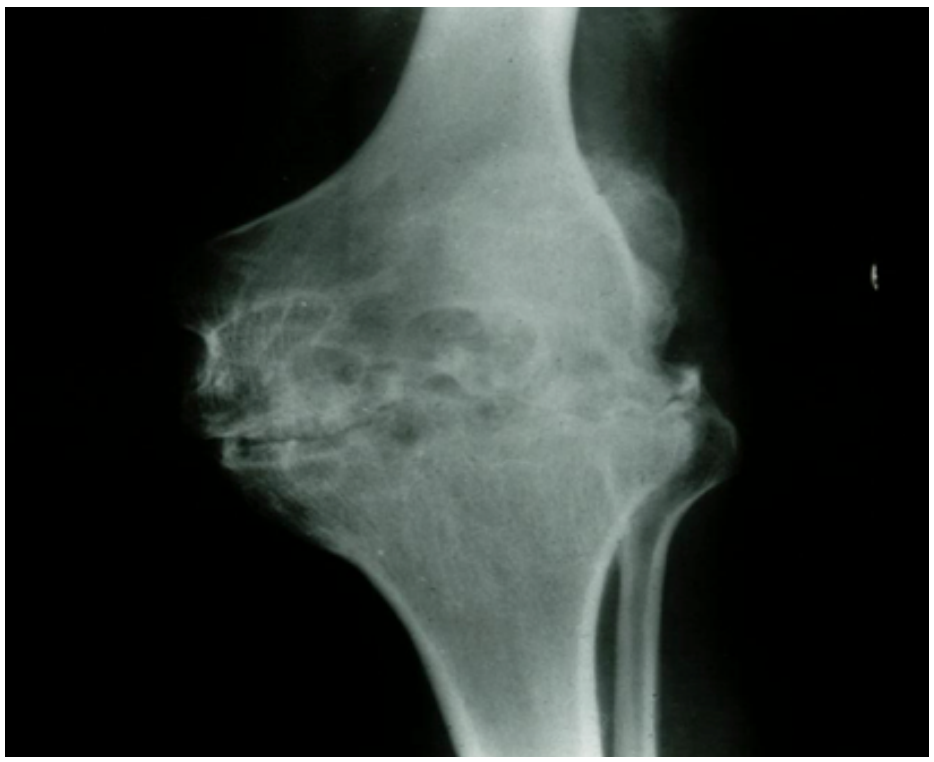

Figure 1: An ankylosed knee joint in a patient with haemophilia. Once a common presentation, with newer and more aggressive treatment this is now quite rare

but working in a dedicated, combined musculoskeletal haemophilia clinic with the patient at the centre of everything we do, can offer great benefits. At the Royal Free, this team consists of:

- Haematologist

Surgeon(s)

- Specialist nurse

- Physiotherapist

Counsellor.

Within the team, we share experience, discuss issues in an open forum and have access to a whole range of skills. Considering what the patients want is at the heart of what we do. Patients want us to talk to each other so they do not feel they are dealing with different people all the time and have to repeat everything. They want us to talk to them in language they understand.

Orthopaedic surgery is extremely complex in haemophilia because we are dealing with bones and with soft tissues. Contractures and muscle wasting can make access to the joint difficult and the end result unpredictable. Joint surgery in a patient with haemophilia is a complex endeavour and can be daunting: excessive bleeding in a normal patient is frightening enough but in a patient with haemophilia it can be very scary. Advanced planning with surgical haemostasis, particularly with our patients with inhibitors, is extremely important. So 


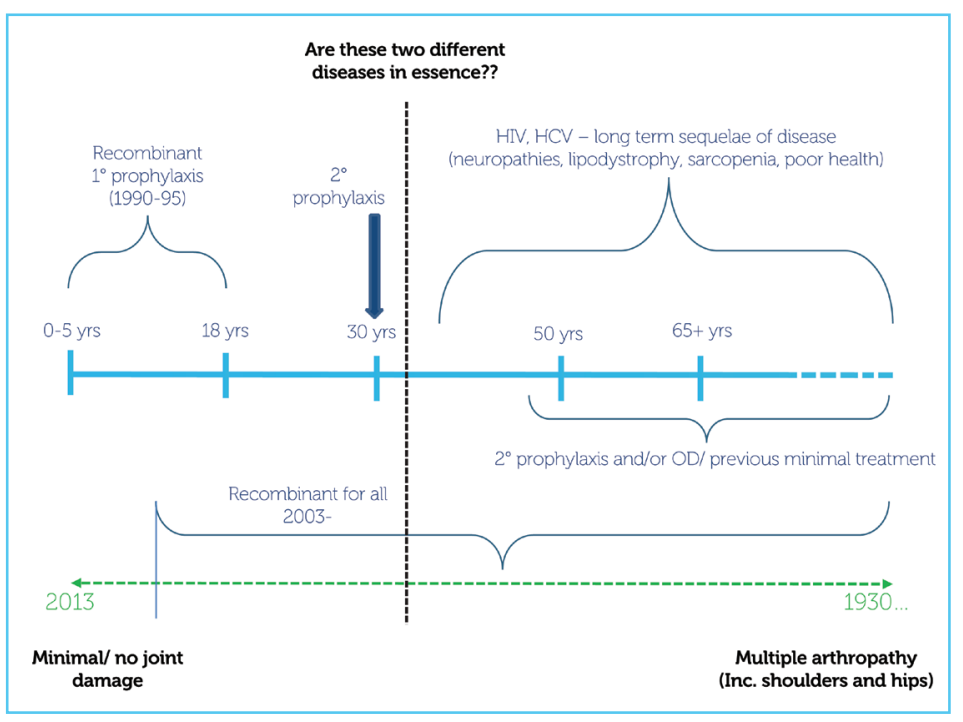

Figure 2: The past history of treatment poses a challenge to the current musculoskeletal management of those with haemophilia

the cornerstone of effective haemophilia treatment is comprehensive care delivered by a multidisciplinary team of diverse specialists and health care professionals.

The goals of orthopaedic surgery in haemophilia are to achieve an accurate diagnosis, exclude infection, achieve effective pain relief, restoration of function, and control of synovitis and prevention of arthritis. We also want to reduce bleeds by early initiation of clotting factor replacement. However, our ultimate goal is to return the patient to independence, which is central to that person's wellbeing.

The cornerstone of management in these patients can be summarised as:

- Synovectomy

- Chemical and radioactive debridements

- Osteotomies

- Arthrodesis

- Prosthetic replacement.

The Royal Free Hospital can boast many important surgical milestones and this is largely down to the foundations laid by Mr Colin Madgwick. From 1945 to the 1960s, orthopaedics was in its infancy and the work consisted mainly of joint aspirations and arthrodeses. Major surgery began in the late 1960s and early 1970s, with the first hip replacement in 1973. Knee replacements began in 1978 and by 2008 the Royal Free Hospital had one of the largest single centre series of knee replacements in the world. Outcomes with total knee replacement in patients with haemophilic arthropathy match those in the nonhaemophilic population [1].

We have assessed why we are so successful and by reviewing papers describing 556 total knee replacements we found that those on high levels of factor for two weeks post-operatively have an infection rate in the region of $2 \%$

[2]. Where factor level replacement was in accordance with the World Federation of Hemophilia recommendations the infection rate was in the region of $9 \%$. We strongly believe this supports the use of a high level clotting factor replacement regime, replacing clotting factors at a higher level for a longer period of time than currently recommended in international guidelines.

In summary, it is the team approach providing comprehensive care that is the way forward in the management of musculoskeletal issues in haemophilia. We start with conservative measures and physical therapies but orthopaedic specialists get involved early on. In this sub-specialty area, we are happy doing joint replacements in younger patients than we would normally consider. But we are aware of the risks of complications and higher infection rates.

\section{The physiotherapist's perspective ...}

From the very beginning, the multidisciplinary team was the model utilised at the Katherine Dormandy Haemophilia Centre. The team includes members from haematology, haemophilia nursing, orthopaedic surgery, physiotherapy and counselling and psychological services, and of course the patient. Over the past 3540 years there has been remarkably little change in the staff in the team. The benefits of this are borne out in the literature that shows that outcomes for patients with musculoskeletal problems in haemophilia is better where there is consistency in the team delivering the care. Musculoskeletal disease management is not just about assessment of a painful knee or having an operation.

It is very much about taking a holistic approach that understands the needs, concerns and even fears of each individual patient. For the patient, the fear of getting a bleed that you know is going to be painful must be horrific. And the worry of being unable to work for a period must be challenging, particularly for those who are self-employed. This is all compounded by the anxiety of coming into hospital, undergoing an intervention and potentially having a general anaesthetic.

We also need to consider the patients' ability to treat themselves, their ability to work, their family history, what their peers think, even the minutiae of their living accommodation. The current cohort of boys coming to the Royal Free from Great Ormond Street are generally able to look after themselves; but some of the men in their 60 s are less able. We have to ask questions about feeding and even going to the toilet. For some, it can be quite difficult to acknowledge that things are not going very well. And the uniqueness of haemophilia is that the mobility issues do not just involve the legs, but potentially all the joints. A patient who is coping well might then have a new baby and a whole new level of mobility is required to cope. These are all issues that sit within the musculoskeletal care remit.

Our patients sit on a spectrum of musculoskeletal disease severity that is dependent on age and the type of treatments available and the issues with that treatments (Figure 2). Young patients now can expect to suffer little or no joint damage but among our oldest patients we see multiple arthropathy involving hips, knees, ankles, 


\section{Haroon Mann}

Haroon Mann is a USA Fellowship Trained Consultant

Trauma \& Orthopaedic Surgeon with a special interest in foot and ankle reconstruction. He has published extensively on bleeding disorders affecting joints, and foot and ankle treatment and management. He has presented at learned societies both nationally and internationally. He is passionate about orthopaedic research and his areas of clinical interest include haemophilic arthropathy and stem cells.

shoulders, backs, small joints and wrists. The two ends of the spectrum are almost two different diseases. And in the middle are the patients of 35 to 40 with HIV, hepatitis C, lipodystrophy and sarcopenia.

\section{Assessments}

Our assessments are regular and thorough. They include a physical examination and a discussion around pain. Pain is generally under-treated in our patients. Often there is an expectation - certainly among elderly patients - that pain is just a side-effect of haemophilia and to be tolerated. Discussions around functional issues are important.

Often patients will not think they have a problem but we will observe something or hear them say something that triggers an alarm. It can be quite difficult to shift a mind set and encourage some patients to acknowledge a problem. Psychological wellbeing is very important in the elderly, a lot of our men live alone, some of them have never married, they have no family, no friends, and so psychologically there are a lot of issues to manage. Patient goals can range on the spectrum from wanting to do BMX-ing, to wanting help with the stairs so they do not have to move house. This is because we are working with youngsters of 13 and 14 all the way through to 83 year olds. It is a challenge because the functional outcomes we can achieve and the patient's expectations can be very different.

\section{The combined clinic}

The combined orthopaedic haemophilia clinic is multidisciplinary. Referrals come direct to physiotherapy where they are triaged and patients may have rehabilitation or go forward to the clinic. Clinics are six- to eight-weekly and led by the consultant orthopaedic surgeon. It is run like a ward round and works very well.

The issues faced by our patients change with age, and may or may not be related to haemophilia but certainly need constant review by the team. For example, a young boy with haemophilia may show $x$-ray evidence of ankle problems that are the consequence not of his haemophilia but of the fact that he is overweight. This is a social problem and much more difficult to treat than his bleeding, requiring a completely different approach from the team. By contrast, we may have an older patient who has shoulders that do not move, knees that are practically
Paul McLaughlin

Paul McLaughlin graduated from the University of Ulster in Belfast in $\mathbf{2 0 0 0}$ and did a Master's in Advanced Physiotherapy (Neuromusculoskeletal Rehabilitation) at King's College London in 2009. He is a member of the Musculoskeletal Association of Chartered Physiotherapists. His clinical interests are management of chronic musculoskeletal pain in haemophilia, lower limb biomechanics and manual therapy and research interest is in patient-focused outcome measures

fused, elbows that are not really functioning as a hinge, and ankles that are very painful and do very little. The options for such a patient may be to do nothing, or to repair or replace the joints. It is a difficult decision that has to be taken with the patient and should encompass their concerns and fears around hospital interventions. Much of this will depend on the patient's previous history and experience of treatment.

From the point of view of integration of roles the physiotherapist, haematologist and orthopaedic surgeon share the same goals - pain relief, restoration of function, reduced bleeding and return or maintenance of independence.

The future

The future in haemophilia holds many challenges. There are young patients with high expectations of what they can do and achieve and older patients for whom medical care and prophylaxis mean they are living longer although their joints do not work very well and they are falling over and may have osteoporosis. We are seeing patients from outside the UK who have not benefited from prophylaxis and treatment and who have significant joint damage at 30 years of age.

In conclusion, we can say that haemophilia is a bleeding disorder that results in musculoskeletal disease. But a patient's pain is individual, just as function is individual and how patients deal with haemophilia is individual. The holistic approach of the multidisciplinary team is the best way to manage all of those issues. And teamwork, communication and collaboration are essential in trying to achieve the best outcomes for our patients.

\section{Disclosures}

The scientific meeting on which this issue of The Journal of Haemophilia Practice is based was sponsored by Baxter, Bayer, CSL Behring, Grifols, Novo Nordisk, Sobi, Pfizer, BPL and Werfen. Editorial support for the article was provided by the publisher.

\section{References}

1. Goddard NJ, Mann HA, Lee CA. Total knee replacement in patients with endstage haemophilic arthropathy -25 year results. J Bone and Joint Surg $\mathrm{Br}$ 2010;92(8):1085-9. doi: 10.1302/0301-620X.92B8.23922.

2. Wong JM, Mann HA, Goddard NJ. Perioperative clotting factor replacement in infected total knee arthroplasty. Haemophilia 2012;18(4):607-12. doi: 10.1111/j.13652516.2011.02728.x 